\title{
Tratamiento ortopédico prequirúrgico de la fisura palatina con la filosoffia de Latham
}

Presurgical orthopedic rreatment of cleft palate with Latham s philosophy

\section{Romero-Maroto, Marlinn* \\ Romance-García, Ana ** \\ Delgado-Muñoz, María Dolores *** \\ Herrero López, Eduardo***}

*Profesor Titular Odontopediatría y Ortodoncia. Universidad Rey Juan Carlos. Madrid.

${ }^{* *}$ Cirujano Maxilofacial. Hospital 12 de Octubre. Madrid.

***Cirujano Pediátrico. Hospital 12 de Octubre. Madrid.
Resumen: El tratamiento ortopédico prequirúrgico de la fisura palatina es un tema controvertido debido a que no está claro si los efectos estéticos son o no duraderos y a que tampoco están claros los efectos que este tratamiento produce sobre la futura oclusión. Existen diferentes modalidades de tratamiento. se presentan dos casos clínicos tratados con la filosofía de Latham.

Palabras clave: Fisura palatina, Ortopedia prequirúrgica, DMA, ECPR.

Abstract: Surgical orthopedic treatment of cleft palate continues to be a controversial theme due to the fact that it is not clear if it has lasting esthetic effects and to the controversy that exists with regard to its effects on dental occlusion. There are different treatment modalities. We present two cases treated with Latham's philosophy.

Key words: Cleft palate, Presurgical orthopedics, DMA, ECPR.

\section{Correspondencia}

Martín Romero Maroto

Corazón de María $84,1^{\circ} \mathrm{E}$.

28002 Madrid.
BIBLID [1138-123X (2005)10:2; marzo-abril 125-240]

Romero-Maroto M, Romance-Carcía A, Delgado-Muñoz MD, Herrero-López E. Tratamiento ortopédico prequirúrgico de la fisura palatina con la filosofía de Latham. RCOE 2005;10(1):199-204. 


\section{Introducción}

Los objetivos del tratamiento ortopédico prequirúrgico en niños fisurados son:

1. Alinear los segmentos y reducir la anchura de la fisura de forma que se reduzca la tensión en los tejidos blandos y se facilite la queiloplastia.

2. Guiar el crecimiento de los segmentos en que se halla dividido el maxilar.

3. Mejorar la función lingual y facilitar la alimentación del neonato.

4. Moldear los cartílagos nasales y reformar la columela ${ }^{1}$.

Sin embargo, la utilización o no de tratamientos ortopédicos previos al cierre labial en los niños fisurados es una de las cuestiones más polémicas en el tratamiento de estos niños. Aunque todo el mundo está de acuerdo en que con la ortopedia prequirúrgica se consigue una mejor estética inicial a nivel labial y nasal ${ }^{2}$, algunos autores ${ }^{3}$ piensan que estos efectos son duraderos, mientras que otros ${ }^{4}$ opinan que no lo son, y que realizar estas técnicas no evita que, cuando el niño crezca, haya que realizar otras cirugías con el fin de mejorar la estética.

También existe controversia en relación a los efectos que estos tratamientos pueden tener sobre la futura oclusión del niño. Mientras que algunos autores ${ }^{5,6}$ piensan que estos tratamientos alteran el crecimiento incrementando la incidencia de maloclusiones sagitales y transversales, otros ${ }^{7 * 8^{* *}}$, piensan exactamente lo contrario.

Respecto al injerto óseo, la única forma de no tener que realizarlo es combinar los tratamientos ortopédicos con la técnica quirúrgica de la gingivoperiosteoplastia ${ }^{9 *}$.

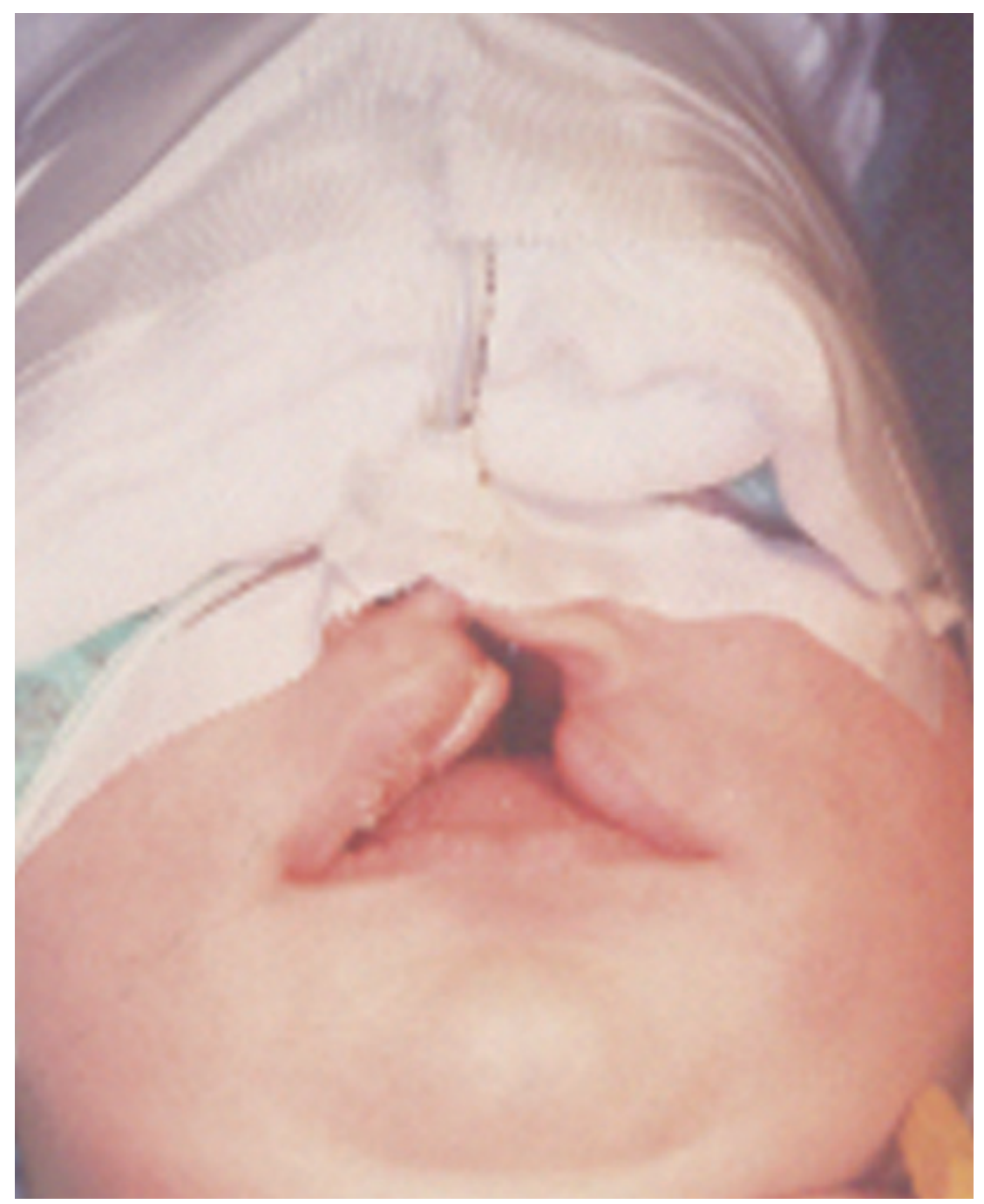

Figura 1. Labio leporino unilateral.

Existen varios métodos de tratamiento ortopédico prequirúrgico:

1. El método de Hotz'10,11, que mediante placas permite dirigir el crecimiento de los fragmentos realizando vaciados y rebases periódicamente. Estas placas pueden ser activas o pasivas $^{12}$.

2. EL método original de McNeil, que para casos bilaterales utiliza placas intraorales y gorros con tracción extraoral13.
3. La técnica de moldeado nasoalveolar descrita por Grayson ${ }^{14}$ y a la que Suri15 ha añadido una pequeña modificación.

4. La técnica de Georgiade-Latham ${ }^{16^{*}}$ que utiliza el DMA (aparato de avance dentomaxilar) para casos unilaterales y el ECPR (aparato de reposición elástica de la premaxila) para casos bilaterales.

Estos aparatos (DMA, ECPR), sujetos por pins, consisten en unas bases de 


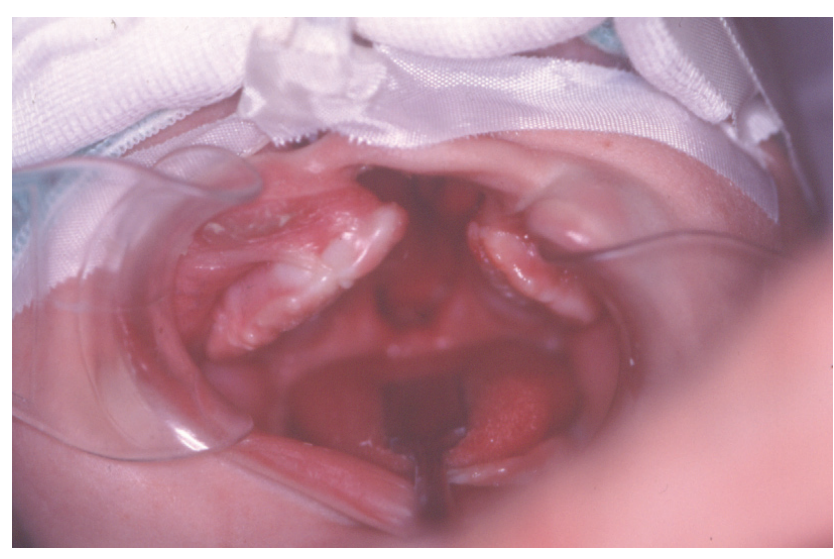

Figura 2. Fisura palatina unilateral con importante separación de los segmentos.

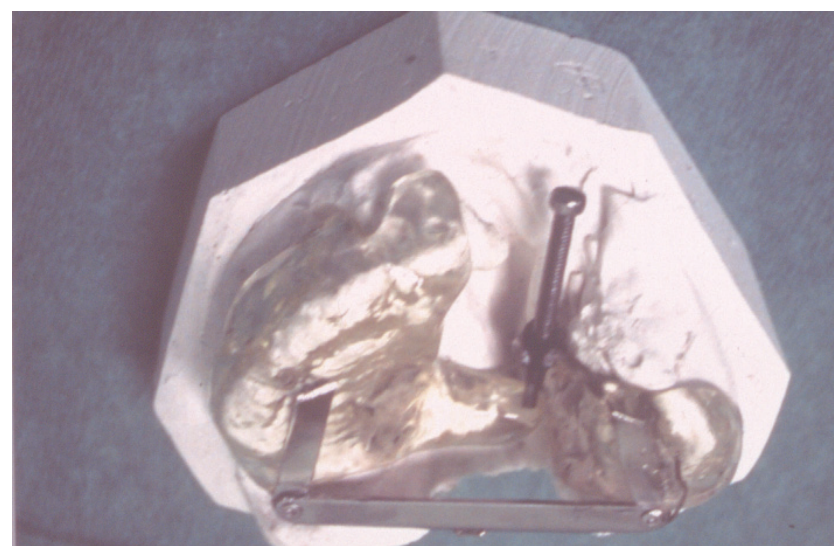

Figura 3. DMA.

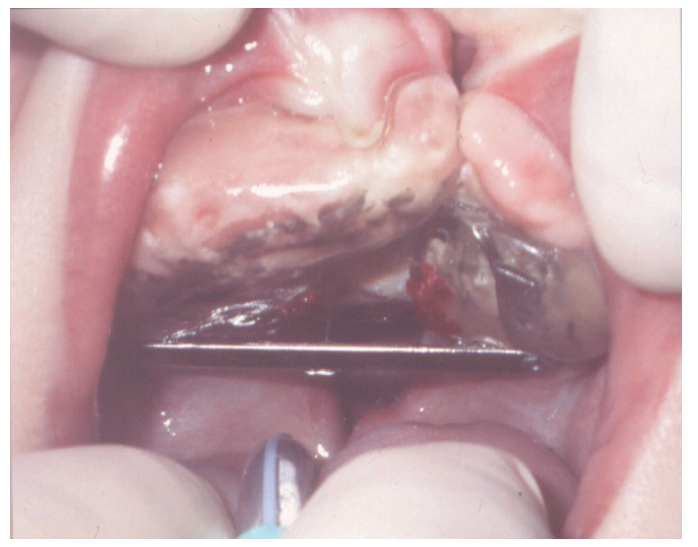

Figura 4. Recolocación de los segmentos y cierre de la fisura con el DMA.

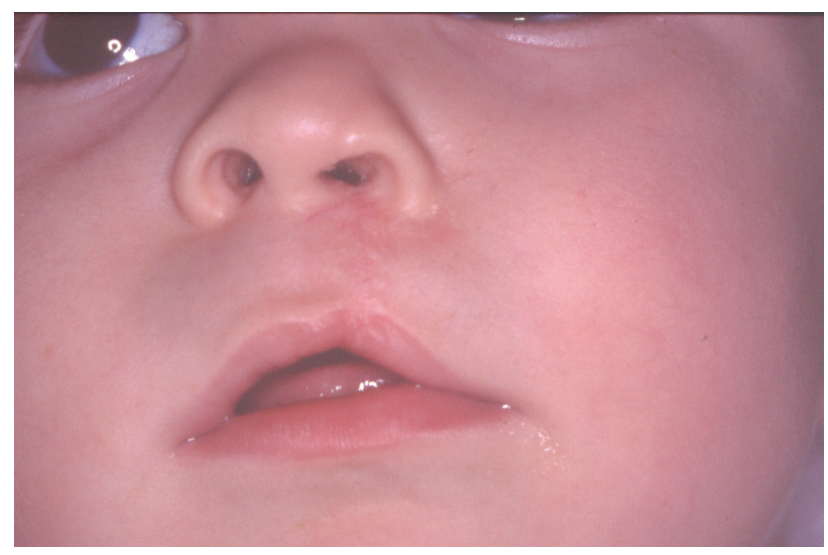

Figura 5. Aspecto del niño tras la queiloplastia. acrílico que se apoyan sobre los segmentos laterales y que están conectados en la parte posterior por un mecanismo de expansión. En los casos bilaterales la retrusión de la premaxila se consigue mediante unas cadenetas que se conectan a un pin que se introduce a través de la premaxila en una posición inmediatamente anterior a la sutura premaxilovomeriana.

En este artículo se presentan dos casos clínicos (una fisura unilateral y una fisura bilateral) tratados con el DMA y el ECPR en la unidad funcional del fisurado del Hospital 12 de Octubre de Madrid.

\section{Caso c「া丨ico 1}

Paciente con labio leporino (fig. 1) y fisura palatina unilateral con una importante separación de los segmentos (fig. 2) que dificultaba la cirugía de cierre labial.

Tras la toma de impresiones con cubetas adecuadas se diseñó un DMA sujetado por cuatro pins (fig. 3) que se colocó bajo anestesia general. Durante cinco semanas los padres activaron el aparato en casa, mientras nosotros revisábamos al niño una vez por semana. Tras lograr realinear los segmentos y cerrar completamente la fisura (fig. 4) se retiró la aparatología y se realizó la queiloplastia con técnica de Malek (fig. 5).

\section{Caso cட́nico ?}

Paciente con labio leporino y fisura palatina bilateral. La premaxila estaba protruida y rotada hacia la derecha, y los segmentos laterales se habían colapsado (fig. 6). Esta protrusión dificultaba la cirugía labial porque aumentaba la tensión de los tejidos blandos.

Tras la toma de impresiones se diseñó un ECPR (fig. 7) que se colo- 


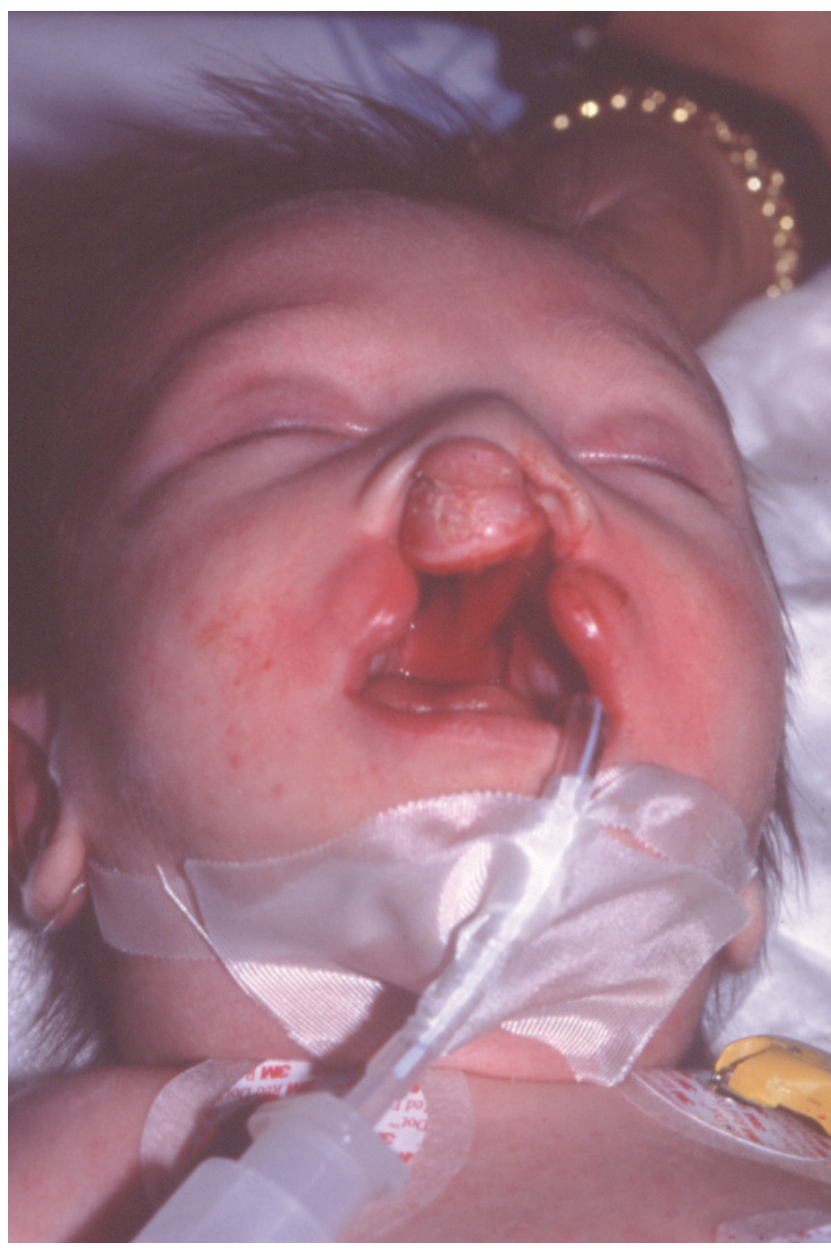

Figura 6. Fisura palatina bilateral con desplazamiento de la premaxila.

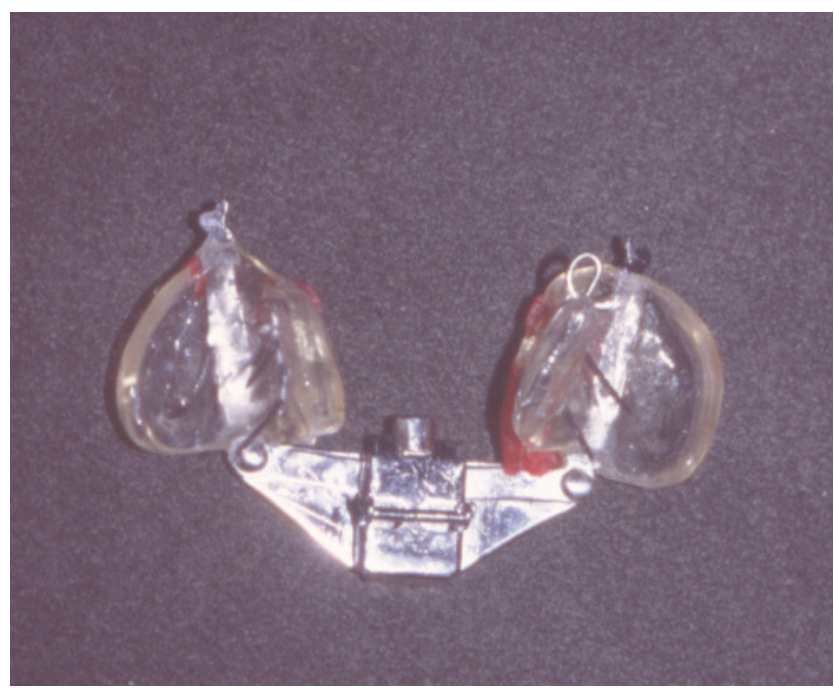

Figura 7. ECPR.

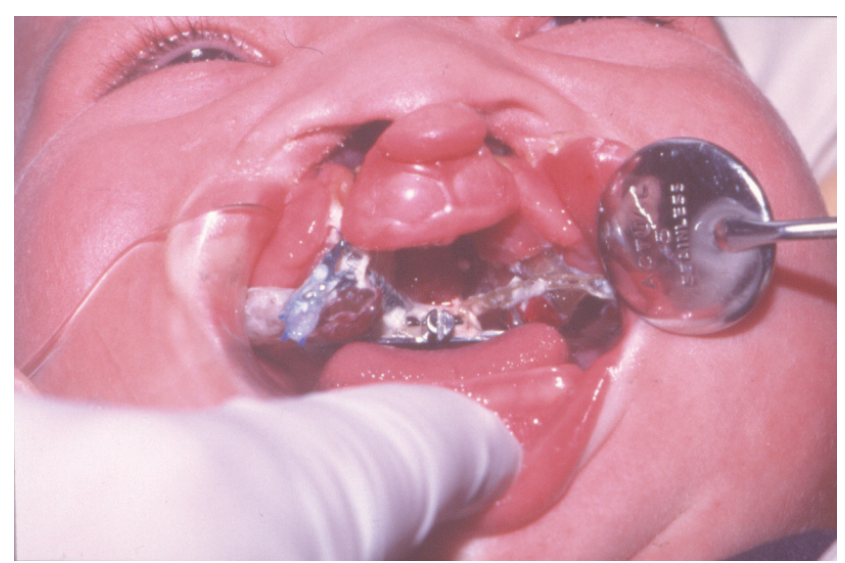

Figura 8. ECPR en boca.

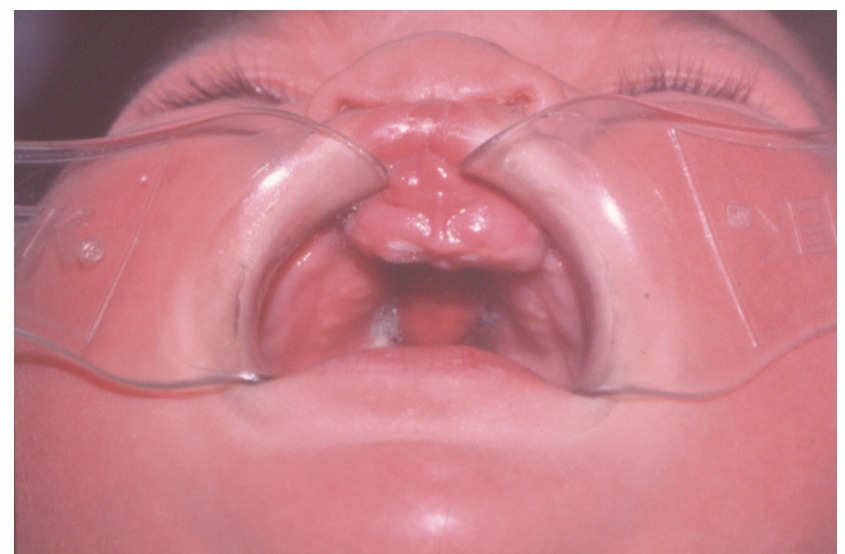

Figura 9. Segmentos alineados con el ECPR.

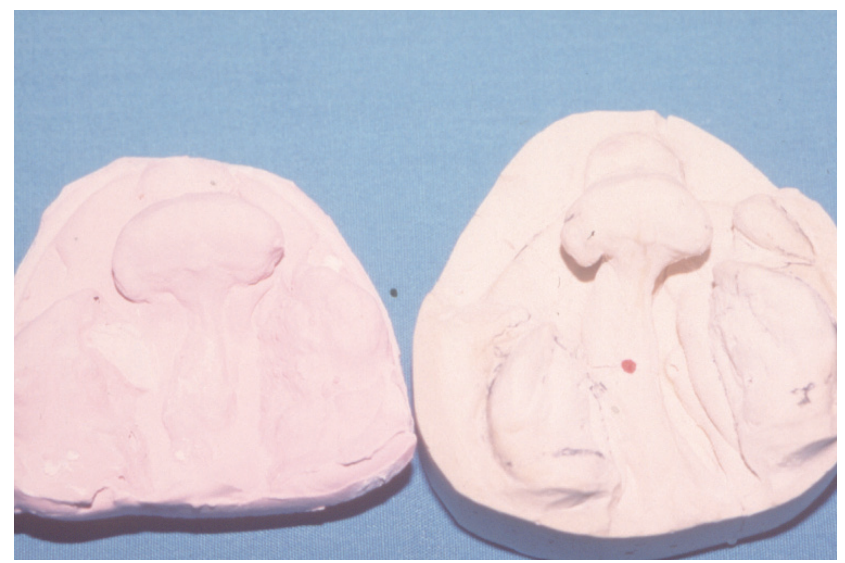

Figura 10. Modelos inicial y final.

RCOE, 2005, Vol 10, N02, 199-204 


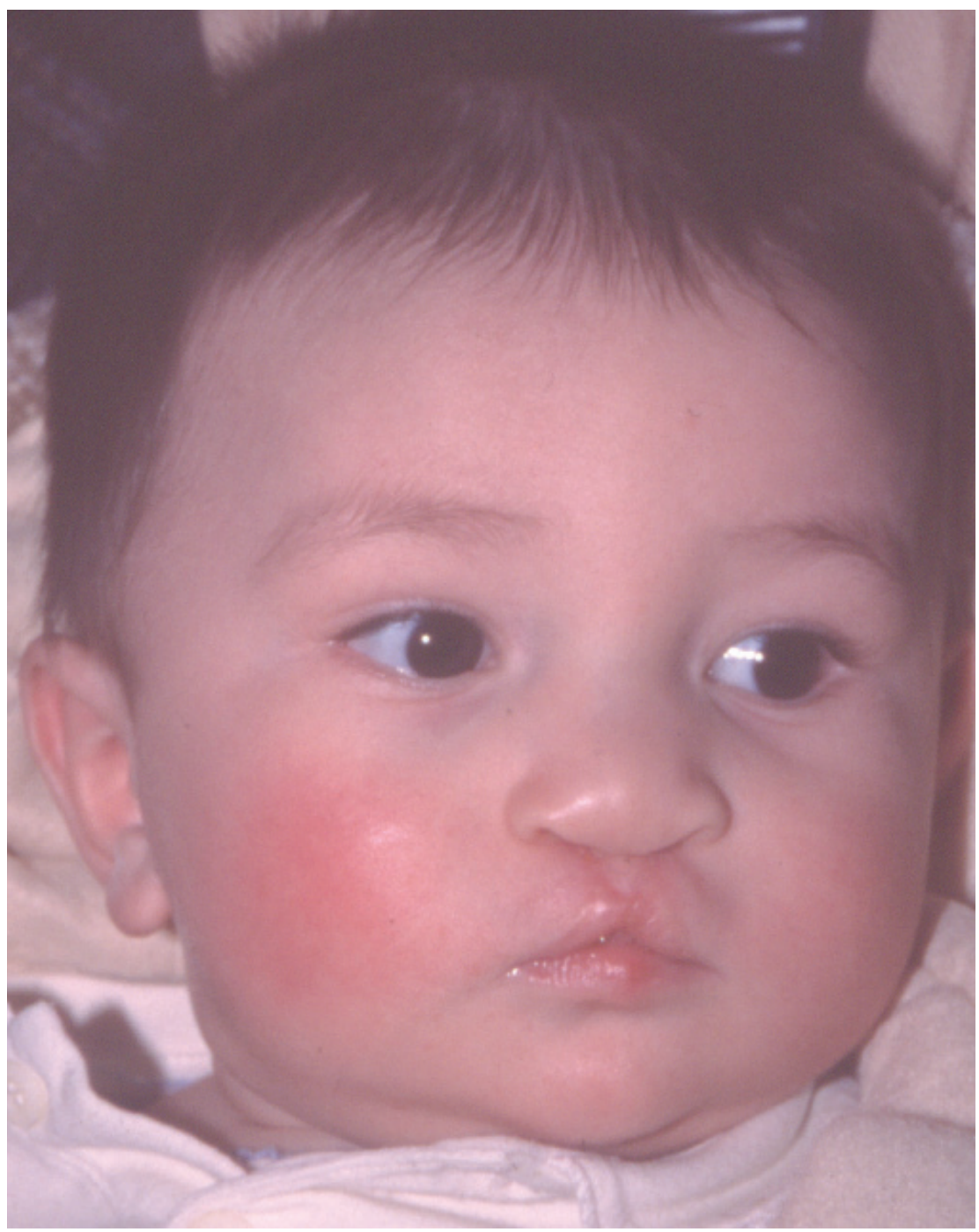

Figura 11. Aspecto del niño tras la queiloplastia.

có bajo anestesia general (fig. 8). Los padres activaban el aparato en casa para lograr la expansión y una vez por semana nosotros modificábamos la tensión de las cadenetas para lograr la retrusión de la premaxila.

Tras seis semanas la premaxila se había retruido y los segmentos esta- ban alineados (figs. 9 y 10) con lo que pudo realizarse la queiloplastia con técnica de Mulliken (fig. 11).

\section{Discusión}

En el tratamiento multidisciplinar de estos casos, se decidió realizar tra- tamiento ortopédico prequirúrgico por la dificultad que para los cirujanos representaba la anchura de la fisura en el caso unilateral y la importante protrusión de la premaxila en el caso bilateral.

Elegimos la técnica de Latham, que es la que habitualmente utilizamos $^{17}$. En el caso unilateral, podríamos haber optado por técnica de Hotz, pero esta técnica, aunque eficaz, requiere más tiempo y exige más cooperación por parte de los padres.

En el caso bilateral existía un problema de espacio, de forma que el espacio existente entre los segmentos laterales no era suficiente para albergar a la premaxila una vez que se retruyera. El ECPR logró la expansión de los segmentos laterales como es habitual en estos $\operatorname{casos}^{18}$.

En este caso se podría haber retruido la premaxila con aparatos extraorales tal como los utiliza Peat ${ }^{19}$, pero aunque estos aparatos son eficaces, si no se manejan muy bien, pueden producir una rotación hacia abajo de la premaxila ${ }^{20}$. Por otra parte la aparatología fija proporciona mejor anclaje que los aparatos removibles cuando es necesario realizar movimientos extensos de los segmentos.

Pese a la controversia que rodea al tratamiento ortopédico prequirúrgico, los resultados en nuestra experiencia, concuerdan con el punto de vista de los autores que defienden que la ortopedia prequirúrgica puede facilitar la queiloplastia en casos complicados. 


\section{Bibliografla recomendada}

Para profundizar en la lectura de este tema, el/los autor/es considera/an interesantes los artículos que aparecen señalados del siguiente modo: *de interés **de especial interés.

1. Romero Maroto M. Tratamiento Ortodóncico de la fisura palatina. En: Bravo González, L.A. Manual de Ortodoncia. Madrid: Editorial Síntesis, S.A, 2003:679-88.

2. Braumann B, Keilig L, Bourauel C, Niederhagen B, Jäger A. 3-Dimensional analysis of cleft palate casts. Ann Anat 1999;181:95-98.

3. Grayson BH, Cutting CB. Presurgical nasoalveolar orthopedic molding in primary correction of the nose, lip and alveolus of infants born with unilateral and bilateral clefts. Cleft Palate Craniofac J 2001;38:193-8.

4. Ross RB, MacNamara MC. Effect of presurgical infant orthopedic on facial esthetics in complete bilateral cleft lip and palate. Cleft Palate Craniofac J 1994;4:68-73.

5. Berkowitz, S. A comparison of treatment results in complete bilateral cleft lip and palate using a conservative approach versus Millard-Latham PSOT procedure. Semin Orthod 1996;2:169-184.

6. Henkel KO, Gundlach KK. What effect does using the Latham devices have on craniofacial growth in uni- and bilateral lip-jaw-palate clefts. Mund Kiefer Gesichtschir 1998;2:55-57

$7 *$. Bitter K. Latham's appliance for presurgical repositioning of the protruded premaxilla in bilateral cleft lip and palate. J Craniomaxillofac Surg1992;20:494-7.

En este artículo se describe el ECPR para pacientes fisurados bilaterales.
8**. Millard DR, Latham R. Cleft lip and palate treated by presurgical orthopedics, gingivoperiosteoplasty and lip adhesion (POPLA) compared with previous lip adhesion method: a preliminary study of serial dental casts. Plast Reconstr Surg 1999;103:1630-44.

Los autores realizan un estudio sobre modelos de forma que puedan analizar los resultados de su técnica.

9*. Santiago PE, Grayson BH, Cutting CB, Gianoutso MP, Brecht LE, Kwon SM. Reduced need for alveolar bone grafting by presurgical orthopedics and primary gingivoperiosteoplasty. Cleft Palate Craniofac. J 1997;35:7780.

Los autores demuestran como con la técnica de la gingivoperiosteoplastia se reduce la necesidad de realizar injertos óseos.

10. Hotz M, Gnoinski W. Comprehensive care of cleft lip and palate children at Zürich University: A preliminary report. Am J Orthod 1976;70:481-504.

11. Hotz M, Gnoinski W, Nussbaumer H, Kistler E. Early maxillary orthopedics in CLP cases: Guidelines for surgery. Cleft Palate Journal 1978;15:405-11.

12. Romero Maroto M, Bravo LA, López Pizarro V, Sánchez del Pozo J. Manejo Clínico del niño con fisura palatina. RCOE 1999;4:241-9.

13. Jones JE, Lynch TR, Sadove AM. Three dimensional premaxillary orthopedic technique for improved position and symmetry prior to cheiloplasty in bilateral cleft lip and palate patients. Quintessence Int 1985;3:229-31.

14. Grayson BH, Santiago PE, Brecht LE, Cutting $\mathrm{CB}$. Presurgical nasoalveolar molding in infants with cleft lip and palate. Cleft Palate Craniofac J 1999;36:486-98.

15. Suri S, Tompson BD. A modified Muscle-Activated Maxillary Orthopedic Aplliance for Presurgical Nasoalveolar Molding in Infants With Unilateral Cleft Lip and Palate. Cleft Palate Craniofac J 2004;41(3):225-9.

16*. Georgiade NG, Latham RA. Maxillary arch alignment in the bilateral cleft lip and palate infant, using the pinned coaxial screw appliance. Plast Reconstr Surg 1975;56:52-60. Los autores describen detalles importantes de la técnica que preconizan.

17. Delgado MD, Marti E, Romance A y cols. Uso de la ortopedia prequirúrgica en pacientes con fisura palatina: Nuestra experiencia. $\mathrm{Cir}$ Pediatr 2004; 17:17-20.

18. Romero M, Latham R, Romance A, Salván R. Treatment of an Infant With a Rare Cleft Resolved With Use of an Orthopedic Appliance. Cleft Palate Craniofac J. 2003;40(6):642-4.

19. Peat JH. Early orthodontic treatment for complete clefts. Am J Orthod 1974;65:28-38.

20. Sierra FJ, Turner C. Maxillary orthopedics in the presurgical management of infants with cleft lip and palate. Pediatr Dent 1995;17:419-23. 